\title{
Adaptive Leader-Follower Coordination of Lagrangian Multi-Agent Systems under Transient Constraints
}

\author{
Christos K. Verginis and Dimos V. Dimarogonas
}

\begin{abstract}
This paper presents a novel control methodology for the coordination of a multi-agent system with 2 nd order uncertain Lagrangian dynamics, while guaranteeing collision and connectivity properties in the transient state. More specifically, we consider that a leader agent aims at tracking a desired pose, while all the agents must avoid collisions with each other. Motivated by cooperative tasks, we also consider that a subset of the initially connected agents must remain connected, in the sense of a connected sensing graph. We employ a key property of the incidence matrix and integrate potential fields with discontinuous adaptive control laws to compensate for unknown dynamic parameters of the model and external disturbances. Simulation results in a realistic dynamics engine illustrate the theoretical findings.
\end{abstract}

\section{INTRODUCTION}

Multi-agent systems are receiving increasing attention during the last decades, due to the advantages they bring with respect to single-agent setups. Their main focus is the design of decentralized control protocols to achieve global tasks, such as consensus, formation, and navigation, while possibly satisfying transient properties, such as connectivity [1]-[7], or collision avoidance [8]-[11].

In the special case of autonomous robotic agents, there has been a lot of research in multi-agent navigation and formation control [8], [9], [12]-[19], while aiming also for inter-agent collision avoidance, which is a crucial safety property of the overall system. Most of these works guarantee the primary agent objectives (navigation or formation) while avoiding collisions from almost all initial configurations, under simplified dynamics (single- and double-integrators). In real scenarios though, potential deviations from the actual agent dynamics might result in failure of meeting the objectives or even cause collisions.

Another important feature of multi-agent systems is their coordination under leader-follower architectures, where an assigned leader aims at executing a task, and the rest of the team is concerned with secondary tasks, such as staying connected with the leader, forming a desired formation, or performing consensus protocols [20]-[23]. Most leader-follower schemes in the related literature consider the follower consensus problem with fixed or time varying communication graphs, where the followers' states converge to the leader's

The authors are with the School of Electrical Engineering and Computer Science, KTH Royal Institute of Technology, SE-100 44, Stockholm, Sweden. Email: $\{$ cverginis, dimos\}akth.se. This work was supported by the H2020 ERC Starting Grant BUCOPHSYS, the European Union's Horizon 2020 Research and Innovation Programme under the GA No. 731869 (Co4Robots), the Swedish Research Council (VR), the Knut och Alice Wallenberg Foundation (KAW) and the Swedish Foundation for Strategic Research (SSF). one, which is assumed to have bounded velocity/acceleration [2], [20]-[24]. Such schemes cannot be extended to multirobot systems though, since collision avoidance is of uttermost importance and it is unreasonable to consider the convergence of the agents' states (e.g., positions) to the same value. Vehicular platoons are special cases of leader-follower structures where collision avoidance is taken into account [25], restricted, however, to the longitudinal platoon-type sensing/communication graph.

Moreover, many of the multi-agent works in the related literature consider simplified/known dynamics (e.g., [4]-[7], [16]-[19]). Such an assumption might have crucial effects on the actual behavior of real robotic systems, whose dynamics are described accurately by Lagrangian models, jeopardizing their performance/safety. More complex/uncertain dynamics are taken into account in [3], [20], [21], without considering collision specifications; [11] integrates collision avoidance with finite boundedness of the inter-agent distances, and [10], [26] deal with the multi-robot collision avoidance problem, without, however, providing theoretical guarantees with respect to the robot dynamics. An MPC methodology is developed in [27], which can be computationally infeasible in real-time when complex dynamics are considered.

In this paper, we propose a decentralized control protocol for the coordination of a multi-agent system with 2nd order uncertain Lagrangian dynamics, subject to collision avoidance and connectivity maintenance. In particular, we consider that a leader agent has to navigate to a desired pose, inter-agent collisions must be avoided, and some of the initially connected agents have to remain connected. By using certain properties of the incidence matrix, we avoid issues of local minima and we relax the assumptions on the connectivity of the graph (as opposed to, e.g., [23], [24]) as well as the access of the leader's velocity by the followers. Moreover, we consider uncertain terms and unknown external disturbances in the dynamic model, which we cope with by using adaptive and discontinuous control laws. Similar adaptive discontinuous schemes are considered in our works [28], which, however, proposes smooth barrier functions for collision avoidance of ellipsoidal agents without, however, guaranteeing achievement of any primary objectives.

The rest of the paper is organized as follows. Section III introduces notation and preliminary background. Section III provides the problem formulation and Section IV discusses the proposed solution. Simulation results are given in Section $\mathrm{V}$ and Section $\mathrm{VI}$ concludes the paper. 


\section{NotATION AND PRELIMINARIES}

The set of natural and real numbers is denoted by $\mathbb{N}$, and $\mathbb{R}$, respectively, and $\mathbb{R}_{\geq 0}, \mathbb{R}_{>0}$ are the sets of nonnegative and positive real numbers, respectively; $\|x\|_{1}$ and $\|x\|$ denote the 1 - and 2-norm, respectively, of a vector $x \in \mathbb{R}^{n}$. The identity matrix is $I_{n} \in \mathbb{R}^{n \times n}$. The open and closed balls with radius $\delta$, centered at $x \in \mathbb{R}^{n}$, are denoted by $\mathcal{B}(x, \delta)$ and $\overline{\mathcal{B}}(x, \delta)$, respectively. The sign function is defined as $\operatorname{sgn}(x)=\{-1,0,1\}$, for $x<0, x=0, x>0$, respectively. The Filippov regularization of a function $f: \mathbb{R}^{n} \rightarrow \mathbb{R}^{k}$ is defined as [29] $\mathrm{K}[f](x):=\bigcap_{\delta>0} \bigcap_{\mu(\bar{N})=0} \overline{\operatorname{co}}(f(\mathcal{B}(x, \delta) \backslash \bar{N}), t)$, where $\bigcap_{\mu(\bar{N})=0}$ is the intersection over all sets $\bar{N}$ of Lebesgue measure zero, and $\overline{c o}(E)$ is the convex closure of the set $E$. The Filippov regularization of $\operatorname{sgn}(x) \in \mathbb{R}$ is denoted by $\mathrm{K}[\operatorname{sgn}](x)=\operatorname{SGN}(x)$ where $\operatorname{SGN}(x)=-1$, if $x<$ $0, \operatorname{SGN}(x)=1$, if $x>0$, and $\operatorname{SGN}(x) \in[-1,1]$, if $x=0$. For a vector $x=\left[x_{1}, \ldots, x_{n}\right]^{\top} \in \mathbb{R}^{n}$, we use $\operatorname{sgn}(x)=\left[\operatorname{sgn}\left(x_{1}\right), \ldots, \operatorname{sgn}\left(x_{n}\right)\right]^{\top} \in \mathbb{R}^{n}$ and $\operatorname{SGN}(x):=$ $\left[\operatorname{SGN}\left(x_{1}\right), \ldots, \operatorname{SGN}\left(x_{n}\right)\right]^{\top} \in \mathbb{R}^{n}$.

Consider the following differential equation with a discontinuous right-hand side:

$$
\dot{x}=f(x, t),
$$

where $f: \mathcal{D} \times\left[t_{0}, \infty\right) \rightarrow \mathbb{R}^{n}, \mathcal{D} \subset \mathbb{R}^{n}$, is Lebesgue measurable and locally essentially bounded.

Definition 1 (Def. 1 of [30]): A function $x:\left[t_{0}, t_{1}\right) \rightarrow$ $\mathbb{R}^{n}$, with $t_{1}>t_{0}$, is called a Filippov solution of 11 on $\left[t_{0}, t_{1}\right)$ if $x(t)$ is absolutely continuous and if, for almost all $t \in\left[t_{0}, t_{1}\right)$, it satisfies $\dot{x} \in \mathrm{K}[f](x, t)$, where $\mathrm{K}[f](x, t)$ is the Filippov regularization of $f(x, t)$.

Lemma 1 (Lemma 1 of [30]): Let $x(t)$ be a Filippov solution of (1) and $V: \mathcal{D} \times\left[t_{0}, t_{1}\right) \rightarrow \mathbb{R}$ be a locally Lipschitz, regular function ${ }^{1}$. Then $V(x(t), t)$ is absolutely continuous, $\dot{V}(x(t), t)=\frac{\partial}{\partial t} V(x(t), t)$ exists almost everywhere (a.e.), i.e., for almost all $t \in\left[t_{0}, t_{1}\right)$, and $\dot{V}(x(t), t) \stackrel{\text { a.e }}{\in} \dot{\widetilde{V}}(x(t), t)$, where $\dot{\tilde{V}}:=\cap_{\xi \in \partial V(x, t)} \xi^{\top}\left[\mathrm{K}[f](x, t)^{\top}, 1\right]^{\top}$, and $\partial V(x, t)$ is Clarke's generalized gradient at $(x, t)$ [30].

Theorem 1 (Corollary 2 of [30]): For the system given in (1), let $\mathcal{D} \subset \mathbb{R}^{n}$ be an open and connected set containing $x=0$ and suppose that $f$ is Lebesgue measurable and $x \mapsto$ $f(x, t)$ is essentially locally bounded, uniformly in $t$. Let $V: \mathcal{D} \times\left[t_{0}, t_{1}\right) \rightarrow \mathbb{R}$ be locally Lipschitz and regular such that $W_{1}(x) \leq V(x, t) \leq W_{2}(x), \forall t \in\left[t_{0}, t_{1}\right), x \in \mathcal{D}$, and $z \leq-W(x(t)), \forall z \in \dot{\widetilde{V}}(x(t), t), t \in\left[t_{0}, t_{1}\right), x \in \mathcal{D}$, where $W_{1}$ and $W_{2}$ are continuous positive definite functions and $W$ is a continuous positive semi-definite on $\mathcal{D}$. Choose $r>0$ and $c>0$ such that $\overline{\mathcal{B}}(0, r) \subset \mathcal{D}$ and $c<\min _{\|x\|=r} W_{1}(x)$. Then for all Filippov solutions $x:\left[t_{0}, t_{1}\right) \rightarrow \mathbb{R}^{n}$ of (1), with $x\left(t_{0}\right) \in \overline{\mathbb{D}}:=\left\{x \in \overline{\mathcal{B}}(0, r): W_{2}(x) \leq c\right\}$, it holds that $t_{1}=\infty, x(t) \in \overline{\mathbb{D}}, \forall t \in\left[t_{0}, \infty\right)$, and $\lim _{t \rightarrow \infty} W(x(t))=0$.

\section{PROBLEM Formulation}

Consider $N>1$ autonomous robotic agents, with $\mathcal{N}:=$ $\{1, \ldots, N\}$, operating in $\mathbb{R}^{n}$ and described by the spheres

${ }^{1}$ See [30] for a definition of regular functions.
$\mathcal{A}_{i}\left(x_{i}\right):=\left\{y \in \mathbb{R}^{n}:\left\|x_{i}-y\right\|<r_{i}\right\}$, with $x_{i} \in \mathbb{R}^{n}$ being agent $i$ 's center, and $r_{i} \in \mathbb{R}_{>0}$ its bounding radius. The agents' motion is described by the Lagrangian dynamics:

$$
M_{i}\left(x_{i}\right) \ddot{x}_{i}+C_{i}\left(x_{i}, \dot{x}_{i}\right) \dot{x}_{i}+g_{i}\left(x_{i}\right)+f_{i}\left(\dot{x}_{i}\right)+d_{i}(t)=u_{i},
$$

where $M_{i}: \mathbb{R}^{n} \rightarrow \mathbb{R}^{n \times n}$ are positive definite inertia matrices, with the property $0<m I_{n} \leq M_{i}(x) \leq \bar{m} I_{n}$, $\forall x \in \mathbb{R}^{n}, i \in \mathcal{N}$, for positive constants $\underline{m}, \bar{m}, C_{i}: \mathbb{R}^{2 n} \rightarrow$ $\mathbb{R}^{n \times n}$ are the Coriolis terms, $g_{i}: \mathbb{R}^{n} \rightarrow \mathbb{R}^{n}$ are the gravity vectors, $f_{i}: \mathbb{R}^{n} \rightarrow \mathbb{R}^{n}$ are unknown vector fields that represent friction-like terms, $d_{i}: \mathbb{R}_{\geq 0} \rightarrow \mathbb{R}^{n}$ are unknown external disturbances and modeling uncertainties, and $u_{i} \in$ $\mathbb{R}^{n}$ are the agents' control inputs, $\forall i \in \mathcal{N}$. The terms $M_{i}$, $C_{i}$ and $g_{i}$ are continuous in their arguments, the terms $f_{i}$ are Lebesgue measurable and locally bounded, and $d_{i}$ are uniformly bounded. Moreover, we consider that the dynamic terms $M_{i}, C_{i}$, and $g_{i}$ include unknown constant dynamic parameters of the agents (e.g., masses, moments of inertia), denoted by the vectors $\theta_{i} \in \mathbb{R}^{\ell}, \ell \in \mathbb{N}, \forall i \in \mathcal{N}$, and satisfy the following well-known properties [31]:

Property 1: The terms $\dot{M}_{i}(x)-2 C_{i}(x, z)$ are skewsymmetric, i.e., $\left(\dot{M}_{i}(x)-2 C_{i}(x, z)\right)^{\top}=2 C_{i}(x, z)-\dot{M}_{i}(x)$ and $y^{\top}\left(\dot{M}_{i}(x)-2 C_{i}(x, z)\right) y=0, \forall x, y, z \in \mathbb{R}^{n}, i \in \mathcal{N}$.

Property 2: The dynamic terms of (2) can be linearly parameterized with respect to the agents' dynamic parameters. That is, for any vectors $x, y, z, w \in \mathbb{R}^{n}$, it holds that $M_{i}(x) y+C_{i}(x, z) w+g_{i}(x)=Y_{i}(y, z, w, x) \theta_{i}, \forall x, y, z, w \in$ $\mathbb{R}^{n}$, where $Y_{i}: \mathbb{R}^{4 n} \rightarrow \mathbb{R}^{n \times \ell}$ are known regressor matrices, and $\theta_{i} \in \mathbb{R}^{\ell}, \ell \in \mathbb{N}$, are vectors of constant but unknown dynamic parameters of the agents, $\forall i \in \mathcal{N}$.

Moreover, we impose the following assumptions on the system (2), which encapsulate standard properties of frictionterms and external disturbances:

Assumption 1: It holds that $\left\|f_{i}\left(\dot{x}_{i}\right)\right\|_{1} \leq f_{b_{i}}\left\|\dot{x}_{i}\right\|_{1}$, $\left\|d_{i}(t)\right\|_{1} \leq d_{b_{i}}, \forall \dot{x}_{i} \in \mathbb{R}^{n}, t \in \mathbb{R}_{\geq 0}$, where $f_{b_{i}}, d_{b_{i}}$ are unknown positive constants, $i \in \mathcal{N}$.

Without loss of generality, we assume that agent $i=1$ corresponds to the team leader, whereas $i>1$ are the followers, which belong to the set $\mathcal{F}:=\{2, \ldots, N\}$. The task of the leader is to navigate to a desired pose $x_{\mathrm{d}} \in \mathbb{R}^{n}$, and the entire team is responsible for guaranteeing collision avoidance as well as connectivity maintenance properties.

In addition, we consider that each agent has a limited sensing radius $d_{\text {con }, i} \in \mathbb{R}_{>0}$, with $d_{\text {con }, i}>\max _{j \in \mathcal{N}}\left\{r_{i}+r_{j}\right\}$, which implies that the agents can sense each other without colliding. Based on this, we model the topology of the multi-agent network through the undirected graph $\mathcal{G}(x):=$ $(\mathcal{N}, \mathcal{E}(x))$, with $\mathcal{E}(x):=\left\{(i, j) \in \mathcal{N}^{2}:\left\|x_{i}-x_{j}\right\| \leq\right.$ $\left.\min \left\{d_{\text {con }, i}, d_{\text {con }, j}\right\}\right\}$, where $x:=\left[x_{1}^{\top}, \ldots, x_{N}^{\top}\right]^{\top} \in \mathbb{R}^{n N}$. Note that, implicitly, $\mathcal{G}$ is time-varying, since $x(t)$ depends on time. We further denote $M(x):=|\mathcal{E}(x)|$. Given the $m$-th edge in the edge set $\mathcal{E}(x)$, we use the notation $\left(m_{1}, m_{2}\right) \in$ $\mathcal{N}^{2}$ that gives the agent indices that form edge $m \in \mathcal{M}(x)$, where $\mathcal{M}(x):=\{1, \ldots, M(x)\}$ is an arbitrary numbering of the edges $\mathcal{E}(x)$. By also denoting $m_{1}$ as the tail and $m_{2}$ as the head of edge $m$, we define the $N \times M$ incidence matrix 
$D(G)=\left[d_{i m}\right]$, where: $d_{i m}=1$, if $i$ is the head of edge $m$; $d_{i m}=-1$, if $i$ is the tail of edge $m$; and $d_{i m}=0$, otherwise. Moreover, we provide the following property of the incidence matrix, which we use in the subsequent analysis:

Property 3: Consider the incidence matrix of a graph $\mathcal{G}$ as $D(\mathcal{G})=\left[d_{1}, \ldots, d_{N}\right]^{\top} \in \mathbb{R}^{N \times M}$, where $d_{i}^{\top} \in \mathbb{R}^{M}$ are the rows of $D$. Then it holds that $\sum_{i \in\{1, \ldots, N\}} d_{i}=0$, i.e., the rows of the incidence matrix sum up to zero.

As discussed before, we also need to guarantee that interagent collisions are avoided for all times, and that some initial edges, denoted by $\mathcal{E}_{0} \subset \mathcal{E}(x(0))$, are preserved. The motivation for that is mainly potential cooperative tasks that the agents have to accomplish, whose details are provided only to a leader agent and hence the graph has to remain connected. Formally, we treat the following problem:

Problem 1: Consider $N$ spherical autonomous robotic agents with dynamics (2). Given Properties 1,2 and Assumption 1. develop a decentralized control strategy that guarantees 1) achievement of the leader's task $\lim _{t \rightarrow \infty}\left(x_{1}(t)-x_{\mathrm{d}}\right)=$ $0,2)$ inter-agent collision avoidance $\mathcal{A}_{i}\left(\begin{array}{l}t \rightarrow \infty \\ x_{i}(t)\end{array}\right) \cap \mathcal{A}_{j}\left(x_{j}(t)\right)=$ $\emptyset, \forall t \in \mathbb{R}_{\geq 0}, i, j \in \mathcal{N}, i \neq j$, and 3 ) connectivity maintenance between a subset of the initially connected agents $\left\|x_{m_{1}}(t)-x_{m_{2}}(t)\right\| \leq \min \left\{d_{\text {con }, m_{1}}, d_{\text {con }, m_{2}}\right\}, \forall t \in \mathbb{R}_{\geq 0}$, $m \in \mathcal{M}_{0} \subset \mathcal{M}(x(0))$, where $\mathcal{M}_{0}:=\left\{1, \ldots, M_{0}\right\}$ is an edge numbering for the edge set $\mathcal{E}_{0}$, with $M_{0}:=\left|\mathcal{E}_{0}\right|$.

\section{MAIN RESUlts}

In this section we propose a decentralized control protocol for the solution of Problem 1

Besides the edge set $\mathcal{E}_{0}$, with edge numbering $\mathcal{M}_{0}$ and $M_{0}$ edges, which needs to remain connected, consider also the complete graph $\overline{\mathcal{G}}:=(\mathcal{N}, \overline{\mathcal{E}})$, with $\overline{\mathcal{E}}:=\{(i, j), \forall i, j \in$ $\mathcal{N}, i<j\}$, the respective incidence matrix $\bar{D}:=D(\overline{\mathcal{G}})$, $\bar{M}:=|\overline{\mathcal{E}}|=\frac{N(N-1)}{2}$, and the edge numbering $\overline{\mathcal{M}}:=$ $\left\{1, \ldots, M_{0}, M_{0}+1, \ldots, \bar{M}\right\}$, where $\left\{M_{0}+1, \ldots, \bar{M}\right\}$ corresponds to the edges in $\overline{\mathcal{E}} \backslash \mathcal{E}_{0}$.

We construct now the local collision and connectivity functions for all edges $\overline{\mathcal{M}}$ and $\mathcal{M}_{0}$, respectively. Given positive constants $\bar{\beta}_{\mathfrak{c}}$ and $\bar{\beta}_{\mathfrak{n}}$, let $\beta_{\mathfrak{c}, m}: \mathbb{R}_{\geq 0} \rightarrow\left[0, \bar{\beta}_{\mathfrak{c}}\right]$ and $\beta_{\mathfrak{n}, l}: \mathbb{R}_{\geq 0} \rightarrow\left[0, \bar{\beta}_{\mathfrak{n}}\right]$, with

$$
\begin{aligned}
\beta_{\mathfrak{c}, m}(x) & :=\left\{\begin{array}{cc}
\vartheta_{\mathfrak{c}, m}(x) & 0 \leq x<\bar{d}_{\mathfrak{c}, m}, \\
\bar{\beta}_{\mathfrak{c}} & \bar{d}_{\mathfrak{c}, m} \leq x
\end{array},\right. \\
\beta_{\mathfrak{n}, l}(x) & :=\left\{\begin{array}{cc}
\vartheta_{\mathfrak{n}, l}(x) & 0 \leq x<\underline{d}_{\mathfrak{n}, l}^{2}, \\
\bar{\beta}_{\mathfrak{n}} & \underline{d}_{\mathfrak{n}, l}^{2} \leq x
\end{array}\right.
\end{aligned}
$$

$\forall m \in \overline{\mathcal{M}}, l \in \mathcal{M}_{0}$, where $\vartheta_{\mathfrak{c}, m}: \mathbb{R}_{\geq 0} \rightarrow\left[0, \bar{\beta}_{\mathfrak{c}}\right], \vartheta_{\mathfrak{n}, l}: \mathbb{R}_{\geq 0}$ $\rightarrow\left[0, \bar{\beta}_{\mathfrak{n}}\right]$ are polynomials that guarantee that $\beta_{\mathfrak{c}, m}$ and $\beta_{\mathfrak{n}, l}$, respectively, are twice continuously differentiable, $\forall m \in \mathcal{M}$, $l \in \mathcal{M}_{0}$. Then, we choose $\beta_{\mathfrak{c}, m}:=\beta_{\mathfrak{c}, m}\left(\iota_{m}\right), \beta_{\mathfrak{n}, l}:=$ $\beta_{\mathfrak{n}, l}\left(\eta_{l}\right)$, where $\iota_{m}:=\left\|x_{m_{1}}-x_{m_{2}}\right\|^{2}-\left(r_{m_{1}}+r_{m_{2}}\right)^{2}, \eta_{l}:=$ $\underline{d}_{\mathfrak{n}, l}^{2}-\left\|x_{l_{1}}-x_{l_{2}}\right\|^{2}, \underline{d}_{\mathfrak{n}, m}:=\min \left\{d_{\text {con }, m_{1}}, d_{\text {con }, m_{2}}\right\}$ and we also set $\bar{d}_{\mathfrak{c}, m}:=\underline{d}_{\mathfrak{n}, m}^{2}-\left(r_{m_{1}}+r_{m_{2}}\right)^{2}, \forall m \in \overline{\mathcal{M}}, l \in \mathcal{M}_{0}$. The terms $\bar{\beta}_{\mathfrak{c}}, \bar{\beta}_{\mathfrak{n}}$ can be any positive constants. Note that $\beta_{\mathfrak{c}, m}$ and $\beta_{\mathfrak{n}, l}$ take into account the limited sensing capabilities of the agents and their derivatives vanish at collisions and connectivity breaks, respectively, of the respective edges. All the parameters for the construction of $\beta_{\mathfrak{c}, m}, \beta_{\mathfrak{n}, l}$ can be transmitted off-line to the agents.

Regarding the uncertain terms of (2), note that $\theta_{i} \in \mathbb{R}^{\ell}$, $f_{b_{i}} \in \mathbb{R}$, and $d_{b_{i}} \in \mathbb{R}$ from Properties 1,2 and Assumption 1 are unknown to the agents. Hence, we define the estimations of these terms $\hat{\theta}_{i} \in \underset{\mathbb{R}^{\ell}}{\widetilde{\theta}_{i}} \hat{f}_{b_{i}} \in \mathbb{R}, \hat{d}_{\underline{b}_{i}} \in \mathbb{R}, \forall i \in \mathcal{N}$, with the respective errors $\widetilde{\theta}_{i}:=\hat{\theta}_{i}-\theta_{i}, \widetilde{f}_{b_{i}}:=\hat{f}_{b_{i}}-f_{b_{i}}, \widetilde{d}_{b_{i}}:=$ $\hat{d}_{b_{i}}-d_{b_{i}}, \forall i \in \mathcal{N}$. In addition, we define the leader error signals $e:=x_{1}-x_{\mathrm{d}}, \phi:=\int_{0}^{t} e(\tau) d \tau$, as well as the combined sliding mode-type error $s_{e}:=e+\lambda \phi$, where $\lambda$ is a nonnegative constant. We also define $\alpha_{i, m}^{\mathfrak{c}}$ and $\alpha_{i, l}^{\mathfrak{n}}$ :

$$
\alpha_{i, m}^{\mathfrak{c}}:=\left\{\begin{array}{ll}
-1, & i=m_{1} \\
1, & i=m_{2} \\
0, & \text { otherw. }
\end{array} \quad \alpha_{i, l}^{\mathfrak{n}} \quad:= \begin{cases}-1, & i=l_{1} \\
1, & i=l_{2} \\
0, & \text { otherw. }\end{cases}\right.
$$

$\forall m \in \overline{\mathcal{M}}, l \in \mathcal{M}_{0}, i \in \mathcal{N}$, which provide boolean values depending on whether agent $i$ is part (head or tail) of edge $m$ and $l$. Finally, we define $\forall m \in \overline{\mathcal{M}}, l \in \mathcal{M}_{0}$, the terms

$$
\beta_{\mathfrak{c}, m}^{\prime}:=\frac{\partial}{\partial \iota_{m}}\left(\frac{1}{\beta_{\mathfrak{c}, m}\left(\iota_{m}\right)}\right), \quad \beta_{\mathfrak{n}, l}^{\prime}:=\frac{\partial}{\partial \eta_{l}}\left(\frac{1}{\beta_{\mathfrak{n}, l}\left(\eta_{l}\right)}\right),
$$

which diverge to infinity in a collision and a connectivity break of the agents $m_{1}, m_{2}$ and $l_{1}, l_{2}$, respectively. We propose now the following decentralized adaptive control protocol. Choose the agents' desired velocity as

$$
\begin{gathered}
v_{d_{1}}=-\left(\gamma_{e}+\lambda\right) s_{e}+k_{1} \sum_{m \in \overline{\mathcal{M}}} \alpha_{1, m}^{\mathfrak{c}} \beta_{\mathfrak{c}, m}^{\prime} \frac{\partial \iota_{m}}{\partial x_{m_{1}}} \\
+k_{1} \sum_{l \in \mathcal{M}_{0}} \alpha_{1, l}^{\mathfrak{n}} \beta_{\mathfrak{n}, l}^{\prime} \frac{\partial \eta_{l}}{\partial x_{l_{1}}} \\
v_{d_{i}}=k_{i}\left(\sum_{m \in \overline{\mathcal{M}}} \alpha_{i, m}^{\mathfrak{c}} \beta_{\mathfrak{c}, m}^{\prime} \frac{\partial \iota_{m}}{\partial x_{m_{1}}}+\sum_{l \in \mathcal{M}_{0}} \alpha_{i, l}^{\mathfrak{n}} \beta_{\mathfrak{n}, l}^{\prime} \frac{\partial \eta_{l}}{\partial x_{l_{1}}}\right),
\end{gathered}
$$

$\forall i \in \mathcal{F}$, that concerns the collision avoidance and connectivity maintenance properties, with the extra term $\left(\gamma_{e}+\lambda\right) s_{e}$ for the leader to guarantee the navigation to $x_{\mathrm{d}}$. The terms $\gamma_{e}$, $k_{i}$ are positive constants, $\forall i \in \mathcal{N}$. Since $v_{d_{i}}$ is not the actual velocity of the agents, we define the errors $e_{v_{i}}:=\dot{x}_{i}-v_{d_{i}}$, $\forall i \in \mathcal{N}$, and design the decentralized control laws

$$
\begin{aligned}
u_{i}= & \sum_{m \in \mathcal{M}} \alpha_{i, m}^{\mathfrak{c}} \beta_{\mathfrak{c}, m}^{\prime} \frac{\partial \iota_{m}}{\partial x_{m_{1}}}+\sum_{l \in \mathcal{M}_{0}} \alpha_{i, l}^{\mathfrak{n}} \beta_{\mathfrak{n}, l}^{\prime} \frac{\partial \eta_{l}}{\partial x_{l_{1}}}-k_{v_{i}} e_{v_{i}}+ \\
& Y_{i}\left(\dot{v}_{d_{i}}, \dot{x}_{i}, v_{d_{i}}, x_{i}\right) \hat{\theta}_{i}-\operatorname{sgn}\left(e_{v_{i}}\right)\left\|\dot{x}_{i}\right\|_{1} \hat{f}_{b_{i}}-\operatorname{sgn}\left(e_{v_{i}}\right) \hat{d}_{b_{i}} \\
& -\widetilde{s}_{e_{i}},
\end{aligned}
$$

$\forall i \in \mathcal{N}$, where $\widetilde{s}_{e_{1}}=s_{e}, \widetilde{s}_{e_{i}}=0, \forall i \in \mathcal{F}$, and $k_{v_{i}}$ are positive gains. Moreover, we design the adaptation signals

$$
\left.\begin{array}{l}
\dot{\hat{\hat{d}}}_{b_{i}}=\gamma_{i, d}\left\|e_{v_{i}}\right\|_{1}, \\
\dot{\hat{f}}_{b_{i}}=\gamma_{i, f}\left\|e_{v_{i}}\right\|_{1}\left\|\dot{x}_{i}\right\|_{1}, \\
\dot{\hat{\theta}}_{i}=-\gamma_{i, \theta} Y_{i}\left(\dot{v}_{d_{i}}, \dot{x}_{i}, v_{d_{i}}, x_{i}\right)^{\top} e_{v_{i}}
\end{array}\right\} i \in \mathcal{N},
$$

with arbitrary bounded initial conditions, and positive constants $\gamma_{i, d}, \gamma_{i, f}, \gamma_{i, \theta}, \forall i \in \mathcal{N}$. Note from (4) that, unlike the 
usual case in the related literature, the leader contributes to the collision avoidance and connectivity maintenance properties, apart from just guaranteeing achievement of its task. Regarding the rest of the terms, $Y_{i}(\cdot) \hat{\theta}, \operatorname{sgn}\left(e_{v_{i}}\right)\left\|\dot{x}_{i}\right\|_{1} \hat{f}_{b_{i}}$, and $\operatorname{sgn}\left(e_{v_{i}}\right) \hat{d}_{b_{i}}$ compensate for the unknown terms $\theta_{i}, f_{b_{i}}$, and $d_{b_{i}}$, respectively, and $e_{v_{i}}$ is a dissipative velocity term that ensures closed-loop stability. The main results of this work are summarized in the following theorem.

Theorem 2: Consider a multi-agent team $\mathcal{N}$, described by the dynamics (2) subject to Properties 112 and Assumption 1. Then, application of the control and adaptation laws (4), (5) with the following choice of control gains:

$$
\begin{aligned}
& \frac{k_{1}(2-a)}{a}-\lambda>\gamma_{e}>k_{1}+\lambda \\
& 2 k_{i}>(1-a)\left(k_{1}+\lambda+\gamma_{e}\right), \forall i \in \mathcal{F},
\end{aligned}
$$

where $a$ is a constant satisfying $a \in\left[0, \frac{k_{1}}{k_{1}+\lambda}\right) \subset[0,1)$, guarantees: 1) navigation of the leader agent to $x_{\mathrm{d}}, 2$ ) connectivity maintenance of the subset $\mathcal{E}_{0}$ of the initial edges, 3) inter-agent collision avoidance, and 4) boundedness of all closed loop signals, from all collision-free initial configurations, i.e., $\mathcal{A}_{i}\left(x_{i}(0)\right) \cap \mathcal{A}_{j}\left(x_{j}(0)\right)=\emptyset, \forall i, j \in \mathcal{N}$, with $i \neq j$, providing thus a solution to Problem 1 . Moreover, it holds that $\lim _{t \rightarrow \infty} v_{i}(t)=0, \forall i \in \mathcal{N}$.

Proof: By employing (2), (4), (5), we can write the closed-loop system as

$$
\dot{\mu} \in f_{\mu}(\mu, t)
$$

where $\mu:=\left[x^{\top}, \dot{x}^{\top}, \hat{d}_{b}^{\top}, \hat{f}_{b}^{\top}, \hat{\theta}^{\top}\right]^{\top}, \dot{x}:=\left[\dot{x}_{1}^{\top}, \ldots, \dot{x}_{N}^{\top}\right]^{\top}$, $\hat{d}_{b}:=\left[\hat{d}_{b_{1}}, \ldots, \hat{d}_{b_{N}}\right]^{\top}, \quad \hat{f}_{b}:=\left[\hat{f}_{b_{1}}, \ldots, \hat{f}_{b_{N}}\right]^{\top}, \quad \hat{\theta}:=$ $\left[\hat{\theta}_{1}^{\top}, \ldots, \hat{\theta}_{N}^{\top}\right]^{\top}$, and $f_{\mu}$ is a set valued function formed by replacing the $\operatorname{sgn}()$ functions with $\operatorname{SGN}()$. Consider the set $\mathcal{X}:=\left\{\mu \in \mathbb{R}^{2 N n+2 N+\ell N}: \mathcal{A}_{i}\left(x_{i}\right) \cap \mathcal{A}_{j}\left(x_{j}\right)=\emptyset, \forall i, j \in\right.$ $\mathcal{N}, i \neq j,\left\|x_{m_{1}}-x_{m_{2}}\right\| \leq \min \left\{d_{\text {con }, m_{1}}, d_{\text {con }, m_{2}}\right\}, \forall m \in$ $\left.\mathcal{M}_{0}\right\}$. Since, initially the agents do not collide and $\mathcal{E}_{0}$ is a subset of the initially connected agents $\mathcal{E}(x(0))$, it holds that $\mu(0) \in \mathcal{X}$. The right hand side of (7) is measurable in $t$ over $\mathbb{R}_{\geq 0}$ and Lebesgue measurable and locally bounded in $\mu$ on $\mathcal{X}$. Therefore, by invoking Prop. 3 of [32], there exists at least a Filippov solution $\mu:\left[0, t_{1}\right) \rightarrow \mathcal{X}$ for some $t_{1}>0$. Consider now the function

$$
V_{1}:=\frac{\lambda^{2}}{2}\|\phi\|^{2}+\frac{1}{2}\left\|s_{e}\right\|^{2}+\sum_{m \in \mathcal{M}} \frac{1}{\beta_{\mathfrak{c}, m}}+\sum_{l \in \mathcal{M}_{0}} \frac{1}{\beta_{\mathfrak{n}, l}}
$$

which is well defined when $\mu \in \mathcal{X}$. We aim to show that $V_{1}$, given its initial boundedness, remains bounded $\forall t \in \mathbb{R}_{\geq 0}$, and so do the terms $\frac{1}{\beta_{\mathfrak{c}, m}}, \frac{1}{\beta_{\mathfrak{n}, l}}$. By considering the time derivative of $V_{1}$, substituting $e=s_{e}-\lambda \phi$ in the term $\lambda^{2} \phi^{\top} e$, and taking into account that $\frac{\partial \iota_{m}}{\partial x_{m_{1}}}=-\frac{\partial \iota_{m}}{\partial x_{m_{2}}}, \forall m \in \overline{\mathcal{M}}$, $\frac{\partial \eta_{l}}{\partial x_{l_{1}}}=-\frac{\partial \eta_{l}}{\partial x_{l_{2}}}, \forall l \in \mathcal{M}_{0}$, we obtain

$$
\dot{V}_{1}=-\lambda^{3}\|\phi\|^{2}+s_{e}^{\top}\left(\dot{x}_{1}+\lambda s_{e}\right)-\beta^{\top}\left(\widetilde{D} \otimes I_{n}\right)^{\top} \dot{x},
$$

where $\beta:=\left[\beta_{\mathfrak{c}}^{\top}, \beta_{\mathfrak{n}}^{\top}\right]^{\top} \in \mathbb{R}^{\bar{M}+M_{0}}, \quad \beta_{\mathfrak{c}}:=\left[\beta_{\mathfrak{c}, 1}^{\prime} \frac{\partial \iota_{1}}{\partial x_{1_{1}}}\right.$, $\left.\ldots, \quad \beta_{\mathfrak{c}, \bar{M}}^{\prime} \frac{\partial \iota_{\bar{M}}}{\partial x_{\bar{M}_{1}}}\right]^{\top} \in \mathbb{R}^{\bar{M}}, \quad \beta_{\mathfrak{n}}:=\left[\beta_{\mathfrak{n}, 1}^{\prime} \frac{\partial \eta_{1}}{\partial x_{1_{1}}}, \ldots\right.$, $\left.\beta_{\mathfrak{n}, M_{0}}^{\prime} \frac{\partial \eta_{M_{0}}}{\partial x_{\left(M_{0}\right)_{1}}}\right]^{\top} \in \mathbb{R}^{M_{0}}$, and $\widetilde{D}:=\left[\bar{D}, D_{0}\right] \in \mathbb{R}^{N \times\left(\bar{M}+M_{0}\right)}$, where $\bar{D}$ and $D_{0}$ are the incidence matrices corresponding to $\overline{\mathcal{E}}$ and $\mathcal{E}_{0}$, respectively. Let now $\widetilde{d}_{i}^{\top} \in \mathbb{R}^{\bar{M}+M_{0}}, i \in \mathcal{N}$, be the rows of $\widetilde{D}$, i.e., $\widetilde{D}=\left[\widetilde{d}_{1}, \ldots, \widetilde{d}_{N}\right]^{\top}$. Then, the last term of (9) can be written as $\beta^{\top}\left(\widetilde{D} \otimes I_{n}\right)^{\top} \dot{x}=\sum_{i \in \mathcal{N}} \beta^{\top}\left(\widetilde{d}_{i} \otimes I_{n}\right) \dot{x}_{i}$ and hence (3) and (4) become

$$
\begin{aligned}
v_{d_{1}}= & -\left(\lambda+\gamma_{e}\right) s_{e}+k_{1}\left(\widetilde{d}_{1} \otimes I_{n}\right)^{\top} \beta \\
v_{d_{i}}= & k_{i}\left(\widetilde{d}_{i} \otimes I_{n}\right)^{\top} \beta, \\
u_{i}= & \left(\widetilde{d}_{i} \otimes I_{n}\right)^{\top} \beta-\widetilde{s}_{e_{i}}+Y_{i}\left(\dot{v}_{d_{i}}, \dot{x}_{i}, v_{d_{i}}, x_{i}\right) \hat{\theta}_{i}-k_{v_{i}} e_{v_{i}} \\
& -\operatorname{sgn}\left(e_{v_{i}}\right)\left\|\dot{x}_{i}\right\|_{1} \hat{f}_{b_{i}}-\operatorname{sgn}\left(e_{v_{i}}\right) \hat{d}_{b_{i}}, \quad \forall i \in \mathcal{N} .
\end{aligned}
$$

Achievement of the desired velocities, i.e., $\dot{x}_{i}=v_{d_{i}}, \forall i \in$ $\mathcal{N}$, implies that $\dot{V}_{1}=-\lambda^{3}\|\phi\|^{2}-\gamma_{e}\left\|s_{e}\right\|^{2}+k_{1} s_{e}^{\top}\left(\widetilde{d}_{1} \otimes\right.$ $\left.I_{n}\right)^{\top} \beta-\sum_{i \in \mathcal{N}} k_{i}\left\|\left(\widetilde{d}_{i} \otimes I_{n}\right)^{\top} \beta\right\|^{2}+\left(\lambda+\gamma_{e}\right) \beta^{\top}\left(\widetilde{d}_{1} \otimes I_{n}\right) s_{e}$. Note that the incidence matrix property (Prop. 3 implies that $\widetilde{d}_{1}=-\sum_{i \in \mathcal{F}} \widetilde{d}_{i}$. By also using the identity $x y=\frac{x^{2}}{2}+\frac{y^{2}}{2}-$ $\frac{1}{2}(x-y)^{2}, \forall x, y \in \mathbb{R}, \dot{V}_{1}$ becomes

$$
\begin{aligned}
\dot{V}_{1} \leq & -\left(\gamma_{e}-k_{1}^{\prime}\right)\left\|s_{e}\right\|^{2}-\left(k_{1}-a k_{1}^{\prime}\right)\left\|\left(\widetilde{d}_{1} \otimes I_{n}\right)^{\top} \beta\right\|^{2} \\
& -\sum_{i \in \mathcal{F}}\left(k_{i}-(1-a) k_{1}^{\prime}\right)\left\|\left(\widetilde{d}_{i} \otimes I_{n}\right)^{\top} \beta\right\|^{2}
\end{aligned}
$$

where $a \in\left[0, \frac{k_{1}}{k_{1}+\lambda}\right), 2 k_{1}^{\prime}:=k_{1}+\lambda+\gamma_{e}$. From (6), we can conclude that $\widetilde{\gamma}_{e}:=\gamma_{e}-k_{1}^{\prime}>0, \widetilde{k}_{1}:=\left(k_{1}-a k_{1}^{\prime}\right)>0$, and $\widetilde{k}_{i}:=k_{i}-(1-a) k_{1}^{\prime}>0, \forall i \in \mathcal{F}$, and the last inequality is non-positive, i.e. $\dot{V}_{1} \leq-\widetilde{\gamma}_{e}\left\|s_{e}\right\|^{2}-\widetilde{k}_{1}\left\|\left(\widetilde{d}_{1} \otimes I_{n}\right)^{\top} \beta\right\|^{2}-$ $\sum_{i \in \mathcal{F}} \widetilde{k}_{i}\left\|\left(\widetilde{d}_{i} \otimes I_{n}\right)^{\top} \beta\right\|^{2} \leq 0$. The actual velocities of the agents, however, are not necessarily equal to the desired ones $v_{d_{i}}$, and therefore we use a backsteppinglike technique to proceed. Consider the vector $\zeta:=$ $\left[\phi^{\top}, s_{e}^{\top}, \sqrt{\beta_{\mathfrak{c}, 1}^{-1}}, \ldots, \sqrt{\beta_{\mathfrak{c}, \bar{M}}^{-1}}, \sqrt{\beta_{\mathfrak{n}, 1}^{-1}}, \ldots, \sqrt{\beta_{\mathfrak{n}, M_{0}}^{-1}}, e_{v}^{\top}, \widetilde{d}_{b}^{\top}, \widetilde{f}_{b}^{\top}\right.$, $\left.\tilde{\theta}^{\top}\right]^{\top} \in \underset{\tilde{d}_{b}}{\mathcal{Z}}$, where $e_{v}:=\left[e_{v_{1}}^{\top}, \ldots, e_{v_{N}}^{\top}\right]^{\top} \in \mathbb{R}^{n N}, \widetilde{d}_{b}:=$ $\left[\widetilde{d}_{b_{1}}, \ldots, \widetilde{d}_{b_{N}}\right]^{\top} \in \mathbb{R}^{N}, \widetilde{f}_{b}:=\left[\widetilde{f}_{b_{1}}, \ldots, \widetilde{f}_{b_{N}}\right]^{\top} \in \mathbb{R}^{N}, \widetilde{\theta}:=$ $\left[\widetilde{\theta}_{1}^{\top}, \ldots, \widetilde{\theta}_{N}^{\top}\right] \in \mathbb{R}^{\ell N}$, and $\mathcal{Z}:=\mathbb{R}^{2 n+2 N+n N+\bar{M}+M_{0}+\ell N}$ Similar to (7), we guarantee the existence of a Filippov solution $\zeta:\left[0, t_{1}\right) \rightarrow \mathcal{Z}$ for the respective closed-loop system obtained by differentiating $\zeta$. We aim to prove that $\zeta(t)$ remains in a compact subset of $\mathcal{Z}$, which implies that $\mu$ remains in a compact subset of $\in \mathcal{X}$. Define the barrier-like function $V: \mathcal{Z} \times\left[0, t_{1}\right) \rightarrow \mathbb{R}_{\geq 0}$, with

$$
\begin{aligned}
V(\zeta, t):= & V_{1}(\zeta)+\sum_{i \in \mathcal{N}}\left\{\frac{1}{2} e_{v_{i}}^{\top} M_{i}\left(x_{i}(t)\right) e_{v_{i}}+\frac{1}{2 \gamma_{i, d}} \widetilde{d}_{b_{i}}^{2}+\right. \\
& \left.\frac{1}{2 \gamma_{i, f}} \widetilde{f}_{b_{i}}^{2}+\frac{1}{2 \gamma_{i, \theta}}\left\|\widetilde{\theta}_{i}\right\|^{2}\right\}
\end{aligned}
$$

for which, by using the fact $\underline{m} \leq M_{i}(x) \leq \bar{m}, \forall x \in \mathbb{R}^{n}$, $i \in \mathcal{N}$, it holds that $W_{1}(\zeta) \leq V(\zeta, t) \leq W_{2}(\zeta)$, where the functions $W_{1}, W_{2}: \mathcal{Z} \rightarrow \mathbb{R}_{\geq 0}$ are defined as $W_{1}(\zeta):=$ $\lambda_{1}\|\zeta\|^{2}, W_{2}(\zeta):=\lambda_{2}\|\zeta\|^{2}$, and $\bar{\lambda}_{1}, \lambda_{2}$ are positive constants. Since initially the agents do not collide and $\mathcal{E}_{0}$ is a subset of the initially connected agents $\mathcal{E}(x(0)), V_{1}$, as defined in (8), is well-defined, and hence $V(\zeta(0), 0), \frac{1}{\beta_{\mathfrak{c}, m}\left(\iota_{m}(0)\right)}, \frac{1}{\beta_{\mathfrak{n}, l}\left(\eta_{l}(0)\right)}$ 
are bounded, $\forall m \in \overline{\mathcal{M}}, l \in \mathcal{M}_{0}$, i.e., $V(\zeta(0), 0) \leq \bar{V}$ for a finite constant $\bar{V}$. By taking the derivative of $V$, and in view of Lemma 11, one obtains $\dot{V}(\zeta(t), t) \stackrel{\text { a.e. }}{\in} \dot{\widetilde{V}}(\zeta(t), t)$. Since $V(\zeta, t)$ is continuously differentiable, the generalized gradient reduces to the standard gradient and one obtains after using $\dot{x}_{i}=e_{v_{i}}+v_{d_{i}}$, 9], and Properties 1, 2,

$$
\begin{aligned}
& \dot{\tilde{V}} \subset-\lambda^{3}\|\phi\|^{2}+s_{e}^{\top}\left(v_{d_{1}}+\lambda s_{e}\right)-\sum_{i \in \mathcal{N}} \beta^{\top}\left(\tilde{d}_{i} \otimes I_{n}\right) v_{d_{i}}+ \\
& s_{e}^{\top} e_{v_{1}}+\sum_{i \in \mathcal{N}}\left\{e _ { v _ { i } } ^ { \top } \left(u_{i}-Y_{i}\left(\dot{v}_{d_{i}}, v_{d_{i}}, \dot{x}_{i}, x_{i}\right) \theta_{i}-f_{i}\left(\dot{x}_{i}\right)-d_{i}(t)\right.\right. \\
& \left.\left.-\left(\widetilde{d}_{i} \otimes I_{n}\right)^{\top} \beta\right)+\frac{1}{\gamma_{i, f}} \widetilde{f}_{b_{i}} \dot{\hat{f}}_{b_{i}}+\frac{1}{\gamma_{i, d}} \widetilde{d}_{b_{i}} \dot{\hat{d}}_{b_{i}}+\frac{1}{\gamma_{i, \theta}} \widetilde{\theta}_{i}^{\top} \dot{\hat{\theta}}_{i}\right\} .
\end{aligned}
$$

Next, by substituting the control laws (10), employing the property $x^{\top} \operatorname{sgn}(x)=\|x\|_{1}, \forall x \in \mathbb{R}^{n}$ (which also implies that $x^{\top} \operatorname{SGN}(x)=\|x\|_{1}$, since $x^{\top} \operatorname{SGN}(x)=\{0\}$ when $x=$ 0 ), as well as (10, and Assumption 11, we obtain

$$
\begin{aligned}
& \max _{z \in \dot{\tilde{V}}}\{z\} \leq-W_{\zeta}(\zeta)+\sum_{i \in \mathcal{N}}\left\{e_{v_{i}}^{\top} Y_{i}\left(\dot{v}_{d_{i}}, \dot{x}_{i}, v_{d_{i}}, x_{i}\right) \widetilde{\theta}_{i}\right. \\
& +f_{b_{i}}\left\|e_{v_{i}}\right\|_{1}\left\|\dot{x}_{i}\right\|_{1}+d_{b_{i}}\left\|e_{v_{i}}\right\|_{1}-k_{v_{i}}\left\|e_{v_{i}}\right\|^{2}+\frac{1}{\gamma_{i, f}} \widetilde{f}_{b_{i}} \dot{\hat{f}}_{b_{i}} \\
& \left.-\left\|e_{v_{i}}\right\|_{1}\left(\left\|x_{i}\right\|_{1} \hat{f}_{b_{i}}+\hat{d}_{b_{i}}\right)+\frac{1}{\gamma_{i, d}} \widetilde{d}_{b_{i}} \dot{\hat{d}}_{b_{i}}+\frac{1}{\gamma_{i, \theta}} \widetilde{\theta}_{i}^{\top} \dot{\hat{\theta}}_{i}\right\}
\end{aligned}
$$

where $W_{\zeta}: \mathcal{Z} \rightarrow \mathbb{R}_{\geq 0}$, with $W_{\zeta}(\zeta):=\widetilde{\gamma}_{e}\left\|s_{e}\right\|^{2}+\widetilde{k}_{1} \|\left(\widetilde{d}_{1} \otimes\right.$ $\left.I_{n}\right)^{\top} \beta\left\|^{2}+\sum_{i \in \mathcal{F}} \widetilde{k}_{i}\right\|\left(\widetilde{d}_{i} \otimes I_{n}\right)^{\top} \beta \|^{2}$. Finally, by substituting $f_{b_{i}}=\hat{f}_{b_{i}}-\widetilde{f}_{b_{i}}, d_{b_{i}}=\hat{d}_{b_{i}}-\widetilde{d}_{b_{i}}, \forall i \in \mathcal{N}$, as well as the adaptation laws [5], we obtain $\max _{z \in \dot{\vec{V}}}\{z\} \leq-W_{\zeta}(\zeta)-$ $\sum_{i \in \mathcal{N}} k_{v_{i}}\left\|e_{v_{i}}\right\|^{2}=:-W(\zeta)$. Therefore, we conclude that $z \leq-W(\zeta), \forall z \in \dot{\tilde{V}}(\zeta(t), t), t \in\left[0, t_{1}\right), \zeta \in \mathcal{Z}$, where $W: \mathcal{Z} \rightarrow \mathbb{R}_{\geq 0}$ is a positive semi-definite function defined on $\mathcal{Z}$. Hence, the conditions of Theorem 1 hold, according to which we conclude that all Filippov solutions starting in $\zeta(0) \in \overline{\mathcal{Z}}:=\left\{\zeta \in \mathcal{B}(0, r): W_{2}(\zeta)<\min _{\|\zeta\|=r} W_{1}(\zeta)\right\}$ are extended to $t_{1}=\infty$, satisfy $\zeta(t) \in \overline{\mathcal{Z}}$ for all $t \in \mathbb{R}_{\geq 0}$ and any positive $r$, and $\lim _{t \rightarrow \infty} W(\zeta(t))=0$. Thus, the terms $\beta_{\mathfrak{c}, m}\left(\iota_{m}(t)\right), \beta_{\mathfrak{n}, l}\left(\eta_{l}(t)\right)$ are bounded, $\forall t \in \mathbb{R}_{\geq 0}, m \in \bar{M}$, $l \in \mathcal{M}_{0}$, which implies that connectivity breaks of the set $\mathcal{E}_{0}$ and inter-agent collisions are avoided, $\forall t \in \mathbb{R}_{\geq 0}$. In addition, it holds that $\lim _{t \rightarrow \infty} e_{v_{i}}(t)=0, \lim _{t \rightarrow \infty}\left(\widetilde{d}_{i} \otimes I_{n}\right)^{\top} \beta(t)=$ $0, \forall i \in \mathcal{N}$, and $\lim _{t \rightarrow \infty} s_{e}(t)=0$, which implies that $\lim _{t \rightarrow \infty} e(t)=0$, since $\lambda>0$, as well as $\lim _{t \rightarrow \infty} v_{i}(t)=0$, $\forall i \in \mathcal{N}$ due to 10 . Note that $r$ can be any positive constant and hence the result is global with respect to $\zeta$, i.e., all collision-free initial configurations that satisfy $\mathcal{E}_{0} \subset \mathcal{E}(x(0))$.

Remark 1: Note that initial connectivity of the graphs $\mathcal{G}(x(0)), \mathcal{G}_{0}$ and connectivity to the leader are not technical requirements, as is usually the case in the related literature (e.g., [23], [24]). Regarding the unknown terms $f_{i}\left(\dot{x}_{i}\right)$, $d_{i}(t), \theta_{i}$, note from Theorem 2 and its proof that these are successfully compensated, without the need of convergence of the respective errors to zero. Regarding the control gains, their selection according to 6 is done off-line. It is worth noting that $a$ determines the degree to which the leader agent participates in the collision and connectivity properties; by selecting $a=0$, the left part of $6 a$ is transformed to $k_{1}>0$ and the right part of $6 \mathrm{~b}$ achieves a maximum with respect to $a$, burdening more the followers. Finally, $\lambda$ can be chosen equal to zero, neglecting the integrator effect (i.e., $s_{e}=e$ ).

\section{SIMULATION RESULTS}

We conducted simulations with $N=6$ UAVs in $\mathbb{R}^{3}$ using the realistic robotic simulator Gazebo [33]. We considered bounding radii $r_{i}=0.35 \mathrm{~m}$, sensing ranges $d_{\mathrm{con}, i}=$ $3 \mathrm{~m}, \forall i \in \mathcal{N}$, and initial positions $x_{1}(0)=[0,0,0.1]^{\top}$, $x_{2}(0)=[2,-0.5,0.1]^{\top}, x_{3}(0)=[-1.5,1.5,0.1]^{\top}, x_{4}(0)=$ $[1,2,0.1]^{\top}, \quad x_{5}(0)=[-1.5,-1,0.1]^{\top}$, and $x_{6}(0)=$ $[0.5,-1.5,0.1]^{\top} \mathrm{m}$. We also considered that the leader has 4 navigation objectives, that is, to sequentially navigate to the points $x_{\mathrm{d}, 1}=[0,0,5]^{\top}, x_{\mathrm{d}, 2}=[4,5,3]^{\top}, x_{\mathrm{d}, 3}=[-2,4,2]^{\top}$, $x_{\mathrm{d}, 4}=[3,-2,3]^{\top} \mathrm{m}$. Since this work provides asymptotic results with respect to the error $e$, the leader switches navigation goal each time it gets closer than $0.075 \mathrm{~m}$ to the current goal, i.e., $\|e\| \leq 0.075 \mathrm{~m}$. We also considered $\mathcal{E}_{0}=\{(1,2),(1,3),(1,4),(3,4),(3,5),(5,6),(2,6)\}$. The unknown parameters $\theta_{i}$ concerned the UAVs' mass and the gravity constant. The control gains and parameters were set as $\lambda=0.00015, \gamma_{e}=0.7, k_{1}=0.5, k_{i}=5$, $\forall i \in\{2, \ldots, 6\}$, which satisfy (6) with $a=0.5$, and $\gamma_{i, \theta}=0.1, \gamma_{i, d}=0.01, \gamma_{i, f}=0.1, k_{v_{i}}=2, \forall i \in$ $\{1, \ldots, 6\}$. The simulation results are shown in Figs. 1. 2 for $t \in[0,277]$ s. More specifically, Fig. 11 shows (a) the evolution of the signal $\left\|s_{e}(t)\right\|+\left\|e_{v_{1}}(t)\right\|$, which converges to zero for each navigation objective, (b) the evolution of the product $\prod_{m \in \overline{\mathcal{M}}} \frac{1}{\beta_{\mathfrak{c}, m}\left(\iota_{m}(t)\right)} \prod_{l \in \mathcal{M}_{0}} \frac{1}{\beta_{\mathfrak{n}, l}\left(\eta_{l}(t)\right)}$, which remains bounded, verifying thus the collision avoidance and connectivity maintenance properties, and (c) the evolution of the products of the adaptation signals $\prod_{i \in\{1, \ldots, 6\}}\left\|\hat{\theta}_{i}(t)\right\|, \quad \prod_{i \in\{1, \ldots, 6\}} \hat{d}_{b_{i}}(t), \quad \prod_{i \in\{1, \ldots, 6\}} \hat{f}_{b_{i}}(t)$, which remain bounded, verifying thus the boundedness of the individual signals. Finally, Fig. 2 shows the control inputs of the UAVs. The simulations were carried out in a ROS-Python interface of an $\mathrm{i} 7-8750 \mathrm{H}$ laptop computer with 12 cores at $2.2 \mathrm{GHz}$ and $16 \mathrm{~GB}$ of RAM and an illustrating video can be found in https: / / youtu . be/bzzXC-v2hEM.

\section{CONCLUSIONS AND FUTURE WORKS}

We presented a decentralized control protocol for the leader-follower coordination of multi-agent systems with connectivity and collision specifications. Future directions will address timed and multi-agent simultaneous navigation.

\section{REFERENCES}

[1] L. Sabattini, C. Secchi, N. Chopra, and A. Gasparri, "Distributed control of multirobot systems with global connectivity maintenance," IEEE Transactions on Robotics, vol. 29, no. 5, pp. 1326-1332, 2013.

[2] M. Ji and M. Egerstedt, "Distributed coordination control of multiagent systems while preserving connectedness," IEEE Transactions on Robotics, vol. 23, no. 4, pp. 693-703, 2007.

[3] C. Sun, G. Hu, L. Xie, and M. Egerstedt, "Robust finite-time connectivity preserving coordination of second-order multi-agent systems," Automatica, vol. 89, pp. 21-27, 2018. 


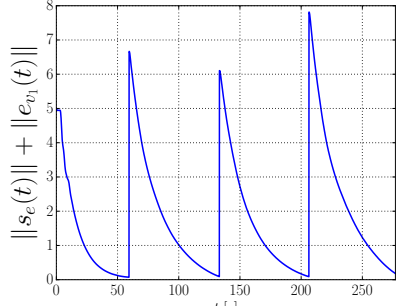

(a)

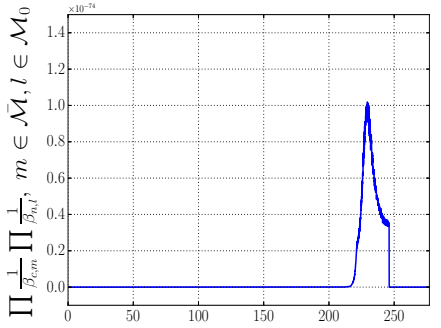

(b) ${ }^{t}[s$

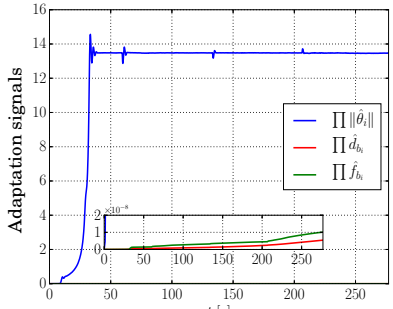

(c)

Fig. 1. (a): The leader signal $\left\|s_{e}(t)\right\|+\left\|e_{v_{1}}(t)\right\| ;$ (b) the product $\prod_{m \in \overline{\mathcal{M}}} \frac{1}{\beta_{\mathfrak{c}, m}\left(\iota_{m}(t)\right)} \prod_{l \in \mathcal{M}_{0}} \frac{1}{\beta_{\mathfrak{n}, l}\left(\eta_{l}(t)\right)}$ (the zero values stem from the computer's lower numerical limits); (c) the adaptation signals $\prod_{i \in\{1, \ldots, 6\}}\left\|\hat{\theta}_{i}(t)\right\|, \prod_{i \in\{1, \ldots, 6\}} \hat{d}_{b_{i}}(t), \prod_{i \in\{1, \ldots, 6\}} \hat{f}_{b_{i}}(t), \forall t \in[0,277] \mathrm{s}$.

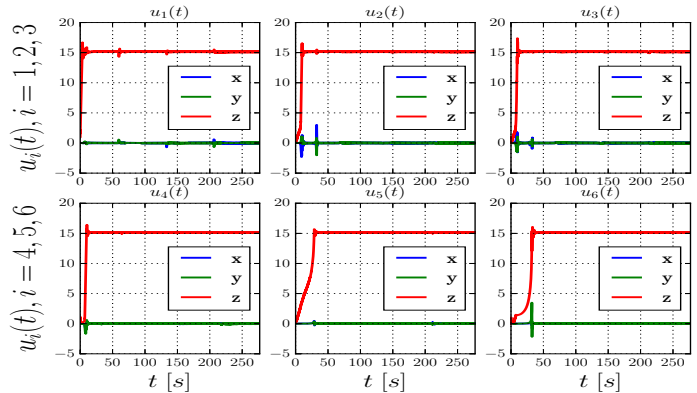

Fig. 2. The resulting control inputs $u_{i}(t), i \in\{1, \ldots, 6\}, t \in[0,277] \mathrm{s}$.

[4] Y. Kantaros and M. M. Zavlanos, "Global planning for multi-robot communication networks in complex environments," IEEE Transactions on Robotics, vol. 32, no. 5, pp. 1045-1061, 2016.

[5] M. Turpin, N. Michael, and V. Kumar, "Capt: Concurrent assignment and planning of trajectories for multiple robots," The International Journal of Robotics Research, vol. 33, no. 1, pp. 98-112, 2014.

[6] M. M. Zavlanos and G. J. Pappas, "Distributed connectivity control of mobile networks," IEEE Transactions on Robotics, vol. 24, no. 6, pp. 1416-1428, 2008.

[7] M. M. Zavlanos and G. J. Pappas, "Potential fields for maintaining connectivity of mobile networks," IEEE Transactions on Robotics, vol. 23, no. 4, pp. 812-816, 2007.

[8] L. Wang, A. D. Ames, and M. Egerstedt, "Safety barrier certificates for collisions-free multirobot systems," IEEE Transactions on Robotics, vol. 33 , no. 3, pp. 661-674, 2017.

[9] L. Sabattini, C. Secchi, and N. Chopra, "Decentralized connectivity maintenance for networked lagrangian dynamical systems with collision avoidance," Asian Journal of Control, vol. 17, no. 1, 2015.

[10] D. Claes and K. Tuyls, "Multi robot collision avoidance in a shared workspace," Autonomous Robots, pp. 1-22, 2018.

[11] S. Li and X. Wang, "Finite-time consensus and collision avoidance control algorithms for multiple auvs," Automatica, vol. 49, no. 11, pp. 3359-3367, 2013.

[12] D. Panagou, "A distributed feedback motion planning protocol for multiple unicycle agents of different classes," IEEE Transactions on Automatic Control, vol. 62, no. 3, pp. 1178-1193, 2017.

[13] C. K. Verginis and D. V. Dimarogonas, "Robust decentralized abstractions for multiple mobile manipulators," IEEE Conference on Decision and Control (CDC), pp. 2222-2227, 2017.

[14] C. K. Verginis, Z. Xu, and D. V. Dimarogonas, "Decentralized motion planning with collision avoidance for a team of uavs under high level goals," IEEE International Conference on Robotics and Automation (ICRA), pp. 781-787, 2017.

[15] C. K. Verginis, A. Nikou, and D. V. Dimarogonas, "Robust formation control in se (3) for tree-graph structures with prescribed transient and steady state performance," Automatica, vol. 103, pp. 538-548, 2019.

[16] H. G. Tanner and A. Kumar, "Towards decentralization of multi-robot navigation functions.," IEEE International Conference on Robotics and Automation (ICRA), vol. 4, p. 4132, 2005.
[17] M. Egerstedt and X. Hu, "Formation constrained multi-agent control," IEEE transactions on robotics and automation, vol. 17, no. 6, 2001.

[18] L. Sabattini, C. Secchi, and C. Fantuzzi, "Arbitrarily shaped formations of mobile robots: artificial potential fields and coordinate transformation," Autonomous Robots, vol. 30, no. 4, p. 385, 2011.

[19] S. Mastellone, D. M. Stipanović, C. R. Graunke, K. A. Intlekofer, and M. W. Spong, "Formation control and collision avoidance for multi-agent non-holonomic systems: Theory and experiments," The International Journal of Robotics Research, vol. 27, no. 1, 2008.

[20] X. Zhang, L. Liu, and G. Feng, "Leader-follower consensus of timevarying nonlinear multi-agent systems," Automatica, vol. 52, 2015.

[21] W. Liu and J. Huang, "Adaptive leader-following consensus for a class of higher-order nonlinear multi-agent systems with directed switching networks," Automatica, vol. 79, pp. 84-92, 2017.

[22] J. Mei, W. Ren, and G. Ma, "Distributed coordinated tracking with a dynamic leader for multiple euler-lagrange systems," IEEE Transactions on Automatic Control, vol. 56, no. 6, pp. 1415-1421, 2011.

[23] T. Gustavi, D. V. Dimarogonas, M. Egerstedt, and X. Hu, "Sufficient conditions for connectivity maintenance and rendezvous in leaderfollower networks," Automatica, vol. 46, no. 1, pp. 133-139, 2010.

[24] Z. Li, X. Liu, W. Ren, and L. Xie, "Distributed tracking control for linear multiagent systems with a leader of bounded unknown input," IEEE Transactions on Automatic Control, vol. 58, no. 2, 2013.

[25] C. K. Verginis, C. P. Bechlioulis, D. V. Dimarogonas, and K. J. Kyriakopoulos, "Robust distributed control protocols for large vehicular platoons with prescribed transient and steady-state performance," IEEE Transactions on Control Systems Technology, vol. 26, no. 1, 2018.

[26] J. Van Den Berg, J. Snape, S. J. Guy, and D. Manocha, "Reciprocal collision avoidance with acceleration-velocity obstacles," IEEE International Conference on Robotics and Automation (ICRA), 2011.

[27] A. Filotheou, A. Nikou, and D. V. Dimarogonas, "Decentralized control of uncertain multi-agent systems with connectivity maintenance and collision avoidance," European Control Conference, 2018.

[28] C. K. Verginis and D. V. Dimarogonas, "Closed-form barrier functions for multi-agent ellipsoidal systems with uncertain lagrangian dynamics," IEEE Control Systems Letters (L-CSS), 2019, Submitted.

[29] B. Paden and S. Sastry, "A calculus for computing filippov's differential inclusion with application to the variable structure control of robot manipulators," IEEE transactions on circuits and systems, vol. 34, no. 1 , pp. 73-82, 1987.

[30] N. Fischer, R. Kamalapurkar, and W. E. Dixon, "Lasalle-yoshizawa corollaries for nonsmooth systems," IEEE Transactions on Automatic Control, vol. 58, no. 9, pp. 2333-2338, 2013.

[31] C. K. Verginis, M. Mastellaro, and D. V. Dimarogonas, "Robust cooperative manipulation without force/torque measurements: Control design and experiments," IEEE Transactions on Control Systems Technology, 2019.

[32] J. Cortes, "Discontinuous dynamical systems," IEEE control Systems, vol. 28 , no. 3,2008

[33] N. P. Koenig and A. Howard, "Design and use paradigms for gazebo, an open-source multi-robot simulator.," IEEE/RSJ International Confernece on Intelligent Robots and Systems (IROS), vol. 4, 2004. 